\title{
Notiz über das Vorkommen alkoholbeständiger karminroter und braunroter Farbstoffe in der Haut von Knochenfischen. \\ Von
}

Professor Dr. med. et phil. E. Ballowitz, Direktor des anatomischen Instituts der Westfälischen Wilhelms-Universität Münster i. W.

(Der Redaktion zugegangen am 11. Juni 1913.)

Es wird allgemein angenommen, daß die in der Haut der Knochenfische vorkommenden, in besonderen Farbstoffzellen, den Erythrophoren, enthaltenen roten Farbstoffe zu der Gruppe der Fettfarbstoffe oder Lipochrome gehören, welche, wie bekannt, leicht in fettlösenden Reagentien gelöst und durch diese, vor allem durch Alkohol, schnell und vollständig aus den Chromatophoren extrahiert werden.

Bei meinen ausgedehnten Untersuchungen ${ }^{1}$ ) über die Chromatophoren und Chromatophoren-Vereinigungen in der Haut der Knochenfische entdeckte ich nun bei bestimmten Teleostiern Farbstoffe, welche sich hiervon abweichend verhalten und in Alkohol beständig sind. Selbst monatelanger Aufenthalt der Hautstücke in starkem Alkohol und in einem Gemisch von Äther sulfur. und absolutem Alkohol zu gleichen Teilen bringen -den Farbstoff nicht zur Auflösung und rufen keine Veränderung an demselben hervor. Diese roten Farb-

1) Vgl. E. Ballowitz, Über chromatische Organe in der Haut von Knochenfischen. Mit 15 mikrophotographischen Abbildungen. Anat. Anz., Bd. 42, Nr. 7/8, 1912. - Derselbe, Die chromatischen Organe in der Haut von Trachinus vipera Cuv. Mit 7 Figuren im Text und Tafeln XIV-XVIII. Zeitschr. f. wissensch. Zoolog.,. Bd. 104, 1913. - Derselbe, Über schwarz-rote, und sternförmige Farbzellen-Kombinationen in der Haut von Gobiiden. Ein weiterer Beitrag zur Kenntnis der Chromatophoren-Vereinigungen bei den Knochenfischen. Mit 5 Tafeln und 25 Textfiguren. Zeitschr. f. wissensch. Zoolog., Bd. 106, 1913. - Derselbe, Über schwarz-rote Doppelzellen und andere eigenartige Chromatophoren-Vereinigungen in der Haut von Knochenfischen. Anatom. Anzeig., Bd. 44, Nr. 5, 1913. - Derselbe, Über die Erythrophoren in der Haut der Seebarbe, Mullus L., und über das Phänomen der momentanen Ballung und Ausbreitung ibres Pigmentes. Nach Beobachtungen an der lebenden Zelle. Mit 2 Tafeln. Archiv für mikroskopische Anatomie, 1913. 
stoffe weichen dadurch von den übrigen, in der Fischhaut sehr verbreiteten roten und gelben Lipochromen ab und müssen eine andere chemische Konstitution besitzen, als diese Lipochrome.

Da hierdurch diese Farbstoffe ein besonderes Interesse beanspruchen, möchte ich die interessierten Fachkreise durch diese Notiz auf sie aufmerksam machen und vielleicht $\mathrm{zu}$ ihrer näheren chemischen Untersuchung anregen.

Die alkoholbeständigen roten Pigmente treten in zwei sehr verschiedenen Farbenniiancen auf und zwar als karminrote und als braunrote.

Der auffälligste Farbenton ist derjenige, welchen ich als den karminroten bezeichnet habe, da er der Karminfarbe am nächsten steht, wenn auch die eigentliche Karminfarbe ein wenig satter ist und etwas ins Bläuliche spielt. In den Präparaten schwankte die Farbe zwischen einem dunkleren, mit einem Stich ins Bläuliche oder Violette gehenden, dem Weinrot sich nähernden Farbenton und mehr helleren, leuchtend roten Farbentönen, die der Malerfarbe Hell-Rosalack nahekommen. Das hängt auch etwas von der Art der Behandlung ab. In den in Balsam eingeschlossenen Präparaten erscheinen diese Chromatophoren leuchtend hell karminrot bis tief weinrot, wenn auch letzteres seltener ist. Diese Farbe weicht sehr ab von dem Rot, Orange und Rotbraun der gewöhnlichen, in Alkohol nicht beständigen Erythrophoren der Fische. Bei den letzteren, z. B. bei den Gobiiden, habe ich höchstens ein leuchtendes Feuerrot angetroffen mit deutlichem Stich in das Gelbliche. Der karminroten Färbung bin ich dagegen bis jetzt nur bei diesen alkoholbeständigen Pigmenten begegnet.

Die karminroten, alkoholbeständigen Pigmentzellen konnte ich bei mehreren Gattungen von Zierfischen feststellen und zwar unter den Cyprinodonten bei Fundulus gularis Boulenger und Fundulus Sjöstedti und bei Haplochilus chaperi Sauvage. Vereinzelt und in kleinen Gruppen traf ich sie auch bei Pantodon Buchholzi Peters an, bei letzterem Fisch, von welchem ich aber nur ein Alkoholexemplar untersuchen konnte, waren sie bisweilen recht groß. 
Besonders schön und zahlreich sind sie bei Fundulus Sjöstedti, bei welchem Knochenfisch ich sie auch zuerst auffand, dann aber auch bei Fundulus gularis; bei beiden Arten zeichnen sich die Männchen durch schöne dunkelrote Flecken und Streifen aus. Auch die Flossen sind mit solchen Flecken und Binden versehen. Alle diese roten Schmuckfarben der Männchen werden bei den beiden Fundulusarten durch die karminroten Erythrophoren erzeugt. Dabei ist hervorzuheben, daß die roten Zellen bei nahe verwandten Arten vermißt werden; so konnte ich sie bei Fundulus chrysotus und Haplochilus rubrostigma nicht auffinden, obwohl der letztere Teleostier, wie schon sein Name sagt, auch leuchtend rote Flecken aufweist. Diese Flecken werden hier aber durch Chromatophoren mit gewöhnlichem, alkohollöslichem, braunrotem Pigment verursacht.

Wie die Untersuchung mit Zeißschen ImmersionsSystemen ergab, ist das karminrote Pigment an gröbere und feinere, in den Erythrophoren in großer Zahl befindliche Körnchen gebunden. Die groben Körner erscheinen rundlich, leuchtend rot, stark lichtbrechend und glänzend; sie sind größer als die Metaninkörnchen der schwarzen Farbstoffzellen, der Melanophoren. Die kleineren Körnchen der karminroten Zellen sind zarter und blasser gefärbt als die großen Körner.

Die andere Farbennuance des alkoholbeständigen Pigmentes ist ein davon recht verschiedenes, helleres oder dunkleres Rotbraun, das sich nicht sehr unterscheidet von dem Farbenton, den die gewöhnlichen, nicht alkoholbeständigen Erythrophoren der Knochenfische unter dem Mikroskope oft darbieten. Ganz besonders dunkel und gesättigt, einem hellen Kaffeebraun sich nähernd, wird die Farbe des zusammengeballten Pigmentes in den lebensfrischen Zellen. Breitet sich das Pigment aus, sodaß es sich in dünner Lage verteilt, so wird die braune Farbe heller und erhält oft, besonders in den Balsampräparaten, einen sehr deutlichen Stich ins Rosa.

So gefärbte Chromatophoren fand ich gleichfalls bei mehreren Zierfischen aus verschiedenen Familien auf und zwar unter den Cyprinodontidae bei Xiphophorus helleri Heckel,

Hoppe-Sey̧ler's Zeitschrift f. physiol. Chemie. LXXXVI. 
218 Ballowitz, Notiz über Farbstoffe in der Haut von Knochenfischen.

unter den Anabantidae bei Betta rubra und unter den Nandidae bei Badis badis.

Auch bei diesen Fischen ist der alkoholbeständige braunrote Farbstoff an kleine Körnchen gebunden.

Die Untersuchung der lebensfrischen Zellen führt nun zu dem bemerkenswerten Resultat, daß die braunroten Körnchen nicht die einzigen Pigmentkörnchen dieser Zellen sind; vielmehr finden sie sich stets vergesellschaftet mit reichlichem gelbem, gleichfalls in kleinsten Körnchen sitzendem Pigment. Wir haben es hier demnach mit einer Vereinigung von braunroten und gelben Farbstoff körnchen in derselben Farbstoffzelle zu tun, und habe ich diese Chromatophoren daher als XanthoErythrophoren bezeichnet. Das Interessante und Eigenartige ist dabei nun, daß der gelbe, in den kleinen Körnchen sitzende Farbstoff zu den Lipochromen gehört, die in Alkohol sehr leicht und vollständig löslich sind. In den in gewöhnlicher Weise hergestellten, zuvor mit Alkohol behandelten Balsampräparaten ist daher von dem gelben Farbstoff keine Spur mehr vorhanden, während die braunroten alkoholbeständigen Farbstoffkörner sich vollständig intakt konserviert haben.

Die obigen Mitteilungen über diese alkoholbeständigen roten Farbstoffe der Fischhaut mögen genügen. Inbetreff alles Näheren über diese auch morphologisch interessanten Chromatophoren verweise ich auf meine soeben im Archiv für mikroskopische Anatomie erschienene ${ }^{1}$ ) ausführliche $\mathrm{Ab}$ handlung; die der Abhandlung beigegebene farbige Tafel illustriert die oben geschilderten abweichenden Farbennuancen.

1) E. Ballowitz, Über Erythrophoren besonderer Art in der Haut von Knochenfischen. Mit Tafel XIV. Archiv für mikroskop. Anatomie, Bd. 82, Abt. I, 1913. Diese Chromatophoren wurden kürzlich von mir auf der 27. Versammlung der Anatomischen Gesellschaft in Greifswald demonstriert. Vgl. die Verhandlungen der Anatom. Gesellsch. auf der 27. Versammlung in Greifswald, 10.-13. Mai 1913. 